\title{
Adenoviruses in Gene Therapy- A Review
}

\author{
Viswanathan.S, Prathiba Srinivasan, Prabhu.S* \\ Department of Biotechnology, Sri Venkateswara College of Engineering, India
}

Copyright (c) 2015 Horizon Research Publishing All rights reserved.

\begin{abstract}
Viruses have become extensively studied over the decade not just for decoding their complex structures but also to understand disease pathways and for the treatment of various diseases as well. The advent of recombinant technology has allowed viruses to be used as vectors in gene therapy, which is the treating of diseases by inserting/deleting genes. One such virus which has risen to prominence because of its flexibility in terms of usage and its versatility in regard to organisms is the adenovirus. This review will elaborate the remarkable progress that has been made with these vectors in the past decade, shortcomings involved in the entire process as well as current biological strategies employed in utilizing adenovirus mediated gene therapy.
\end{abstract}

Keywords Adenovirus, Diseases, Recombinant Technology and Gene Therapy

\section{Introduction}

Adenoviruses are icosahedral viruses of size 90 to $100 \mathrm{~nm}$. Their structure is composed of 252 capsomeres with 240 hexons and 12 pentons at the vertices of the icosahedral. The adenoviral capsid contains at least 13 proteins. Each hexon consists of a polypeptide trimer required for assembly of the virus particles and the penton base consists of a pentamer of polypeptides. A fibrous protein extends out from the vesicles of the penton. It is known to play a role in the recognition and binding of the virus to cell receptors [1]. The structure of adenovirus is shown in Figure 1 below.

\section{Adenovirus Genome}

The adenovirus genome consists of linear, non-segmented double stranded DNA ranging between 26 and $45 \mathrm{Kbp}$ and consists of about 22-40 distinct genes [2]. Its genome is significantly larger than that of other viruses within its group, group I, yet it is still considered a relatively "simple virus" and is extremely dependent on host cell for life cycle and replication processes [2]. The DNA has a terminal $55 \mathrm{kDa}$ protein within each of its 5 ' ends of its linear dsDNA which serve as primers in viral replication and ensure that all of the viral genome is replicated [2]. The terminal sequences of each DNA strand present on 5' and 3' ends are inverted repeats (approximately $100 \mathrm{kbps}$ ). Hence upon denaturation, the single strand has a "panhandle" structure- which allows for DNA replication.

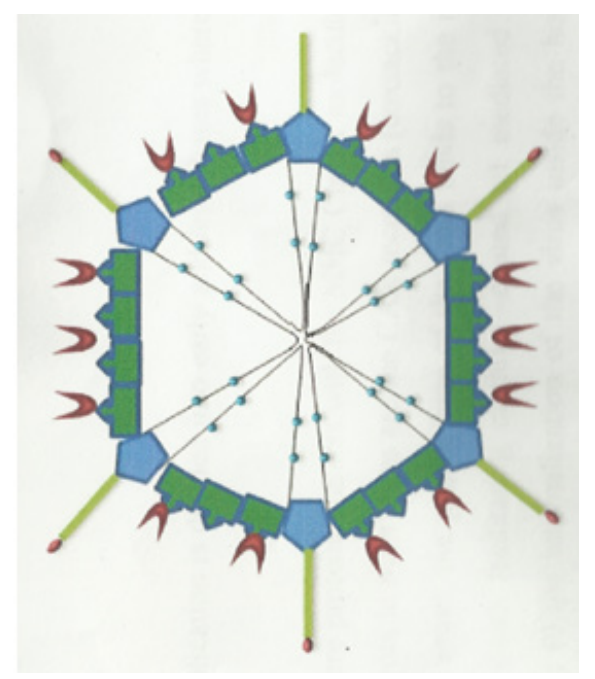

Figure 1. Adenovirus structure.

\section{Replication of Genome}

Adenoviral replication is divided into early and late phases where the DNA replicates in the late phase.

Initially, the fibre protein interacts with the MHC Class I molecule on the cell's surface. The host cell receptors in most cases are the CAR receptors (coxsackie and adenovirus receptor). Following this event, the penton base of the virus binds to the integrins, which behave as secondary receptors, facilitating clathrin-coated pit mediated endocytosis on the cell surface leading to the internalization of the virus inside the host cell. The virion is now present inside a phagocytic vacuole. Toxic action of the pentons causes rupture of the vacuole releasing the virus into the cytoplasm. This is followed by the cytosolic transport to the nucleus transportation by the interaction between hexon and microtubules. Uncoating follows after which the virus core enters the host cell nucleus. The viral DNA is released and undergoes replication. Multiple virus copies are produced and after certain duration of infection, cell lysis occurs and virus copies are produced. The process of viral DNA replication is shown in Figure 2 below. 


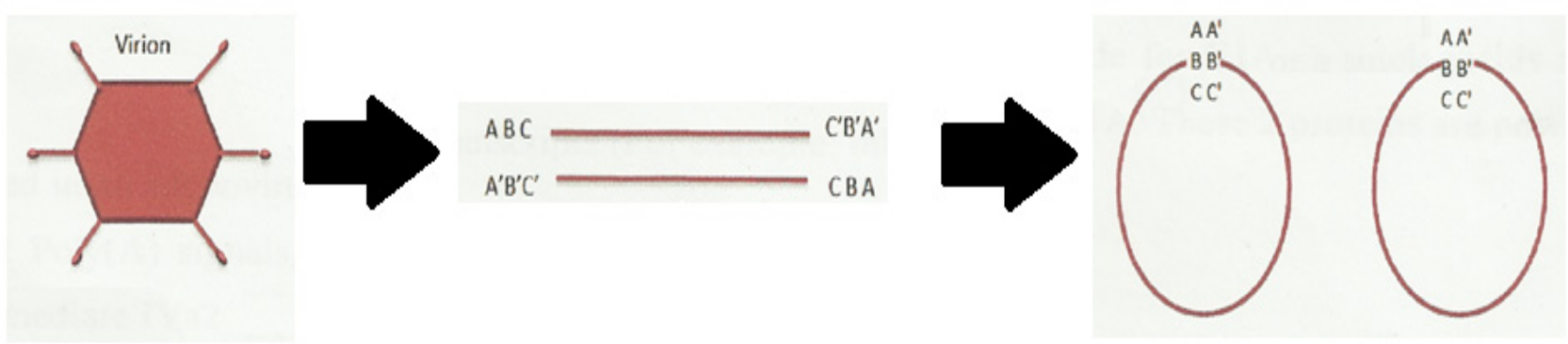

Figure 2. Viral DNA replication.

Adenoviruses also interact with several other viruses like parvoviruses (Adeno-associated virus), SV40 (polyomavirus, which acts as a helper virus for co-infection and in penetrating host cells).

\section{Expression of Genome}

Around 50 genes get expressed in an adenovirus genome. The entire framework consists of 8 transcriptional promoters, 12 Poly(A) signals, 6 early transcription units- E1A, E1B, E2A, E2B, E3 and E4, 2 intermediate Iva2 and IX. The late genes which participate in transcription are L1, L2, L3, L4 and L5. For a single common promoter there are different pol (A) signals each giving rise to different transcripts (For example: mRNA1 and mRNA2).

The E1A gene E1A induces cells into S phase. Hence it is crucial for viral replication. Two mRNAs exist that encode for E1A. First, one yields a 289 a/a long E1A and the second one yields a 243 a/a long E1A [3]. These two proteins are powerful transcriptional activators for several viral and cellular genes. Figure 3 shows the adenovirus transcription.

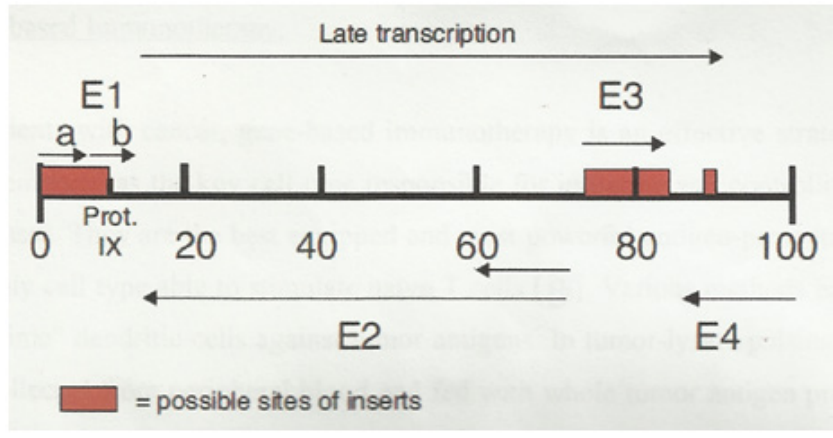

Figure 3. Adenoviral transcription

\section{Pathogenesis}

Adenoviruses cause common infections in the respiratory, gastrointestinal tracts and the eye. Usually, infants fall risk to pharyngitis, pneumonia etc., caused by adenoviruses [4].

\section{Clinical Applications of Gene Therapy with Adenoviral Vectors}

Adenoviruses are highly effective in gene therapy for their ability to efficiently transduce cells that are both dividing and non-dividing [5]. They also have the capacity to hold large segments of DNA (upto $7.5 \mathrm{kbps}$ ) as they are easily manipulated with recombinant DNA techniques and have the ability to produce high titers [5]. The process involves the injection of a new gene into an adenoviral vector, which is further allowed to inject the gene in a human cell. The new gene will thus make a functional protein which was previously defective [6]. A diagrammatic representation is given in figure 4 below.

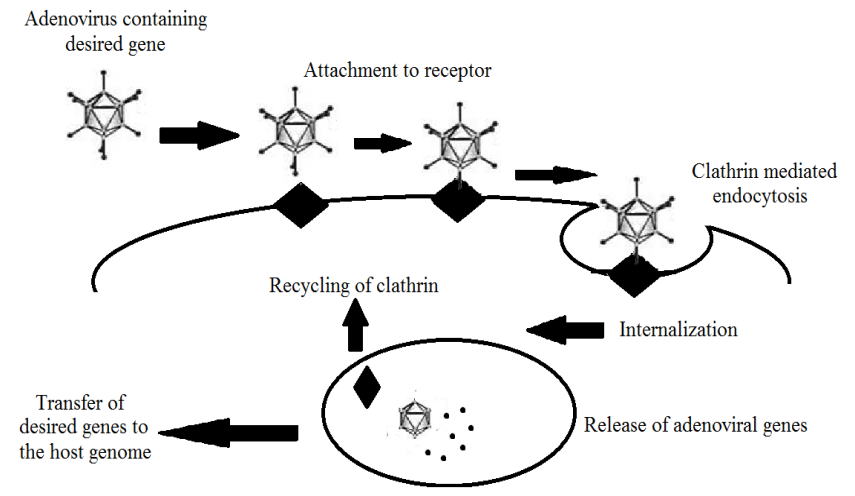

Figure 4. Adenovirus mediated gene transfer

Today, adenoviral vectors are used in suicide gene therapy, in gene-based immunotherapy, in gene replacement strategies, and in approaches that combine gene therapy with chemotherapy. In addition, treatment with replication competent adenovirus vectors is showing promising results in clinical trials.

\section{Suicidal gene therapy}

The fascinating concept of suicide gene therapy (also known as prodrug therapy) refers to the delivery into tumor cells of enzymes that metabolize systematically administered nontoxic prodrugs to locally active chemotherapeutic agents. Treatment efficacy is enhanced by the death of neighboring, nontransduced cells (bystander effect) [7]. In studies done with different animals, adenoviral delivery of the herpes simplex virus thymidine kinase (HSV-tk) gene, which activates the prodrug ganciclovir, has been one of the most effective approaches in treating experimental brain tumors. Recent phase I clinical trials, however, showed substantial toxicity at high levels of vector particles due to a multifactorial cellular and humoral immune response to the first-generation vectors [8]. 


\section{Gene-based Immunotherapy}

In patients with cancer, gene-based immunotherapy is an effective strategy. Dendritic cells have emerged as the key cell type responsible for initiating and controlling cellular immune responses. They are the best equipped and most powerful antigen-presenting cells (APC) and the only cell type able to stimulate naïve $\mathrm{T}$ cells [9]. Various methods have been developed to "prime" dendritic cells against tumor antigens. In tumor-lysate pulsing, the dendritic cells are collected from peripheral blood and fed with whole tumor antigen proteins from lysates. The dendritic cells take up and process the proteins, then present their antigenic epitopes through the exogenous MHC class II pathway and in MHC class I molecules. Another method that proved to protect mice from tumor spread was fusion of dendritic cells with tumor cells. This enabled the tumor cell to present antigens in the manner of an APC [10].

Another approach to the production of patient-specific vaccines involves transducing autologous tumor cells so that they secrete GM-CSF. The clinical potential of this approach has been demonstrated in metastatic melanoma, and in May 2001, a phase II study of vaccination with adenoviral vector in patients with non-small cell cancer (NSCLC) was underway [11].

\section{Gene Replacement and Combinations of Gene Therapy with Standard Antitumor Strategies}

Roth et al. showed that the delivery of wild-type $p 53$ to cells with $p 53$ mutations increased their radiation sensitivity [12]. Tumor cells expressing wild-type $p 53$ are more sensitive to chemotherapeutic agents and radiation than are cells that lack functional $p 53$. The heightened sensitivity of cells with wild-type $p 53$ is thought to be attributable to their propensity to undergo $p 53$-mediated apoptosis (programmed cell death) blocking of the apoptosis pathway are often associated with resistance of cancer cells to current antitumor treatment strategies. Gene therapy has the potential to overcome this block by re-establishing apoptosis-inducing regulators (E2F-1, wild-type $p 53$, interleukin-6 [IL-6], interferon- $\alpha$, Bax) or by transferring genes that induce cell suicide directly (e.g., FAS-ligand, Caspase-8, tumor necrosis factor- $\alpha$ ) [13]. In animal models, treatment with a combination of cisplatin and adenovirus- $p 53$ led to enhanced apoptosis and suppression of tumor growth [14]. We have found increased apoptosis cell death in breast cancer cell lines treated with a combination of adenoviral vectors with the E2F-1 transcription factor gene plus paclitaxel and doxorubicin indicating a strong synergistic effect [15]. Chemotherapy, in return, enhances expression of transduced genes from adenoviral vectors with a wide range of promoters, and radiation improves immediate transduction efficiency and duration of transgene expression [16].

\section{Treatment with Replication-Competent Adenoviral Vectors}

Treatment with replication-competent adenoviral vectors specific to the target tissue has been considered as a means of enhancing the transduction and expression rate of adenoviral vectors. The major advantage of these cytolytic vectors is their potential to reach widespread metastases. Delivery of replication-competent vectors in combination with incorporated suicide genes or prodrugs could reduce systemic cytotoxic effects are inflammatory responses [17].

\section{Clinical Trials of Gene Therapy with Adenoviral Vectors}

There has been an increase in the number of clinical trials based on gene therapy strategies. Over the last three years, the number of gene therapy trials targeting cancer has increased by about $50 \%$ each year. The phase I trials are designed to evaluate the toxicity of treatment. The on-going phase II clinical trials of adenoviral gene therapy illustrate the growing variety of transgenes and methods of action that can be used in gene therapy. This variety underscores the exceptional flexibility of adenoviral vectors. Expression of the therapeutic gene decreases with increasing distance from the injection site, thus there is a need for more advanced vector targeting that allow for systematic application of the vectors [18].

\section{Improved Adenoviral Vector Systems}

The combination of adenoviral vectors with others viral vectors and the targeting of adenoviral vectors to the tumor cells with controlled expression of the therapeutic transgene have reached the preclinical stage. These improvements finally will allow us to exploit the whole potential of gene therapy.

\section{"Gutless" or "helper-dependent" adenoviral vectors}

Recently, "gutless" adenoviral vectors - vectors that are devoid of all viral-protein-coding DNA sequences-have been developed [19]. In this helper-dependent vector system, one vector (the helper) contains all the viral genes required for replication but has a conditional gene defect in the packaging domain making it less likely that its DNA is packaged into a virion. The second vector contains only the ends of the viral genome, therapeutic gene sequences, and the normal packaging recognition signal, which allows this further reduced toxicity and prolonged gene expression in animals, this helper-dependent system allows the introduction of up to $32 \mathrm{~kb}$ of foreign DNA [20]. Processing of gutless adenoviral vectors is currently labor intensive, but it is anticipated that the adaptation of antibody-based purification systems that use affinity chromatography can further enhance the purity of vectors and their large-scale fabrication [21].

\section{Hybrid Vector Systems}

Hybrid vector systems that combine the highly efficient infection capacity of adenoviruses with the long-term genomic-integrating potential of retroviruses and adeno-associated viruses are currently being tested. Such hybrid systems showed efficacy in murine cancer models [22]. 
Proof of the great potential of vector engineering is the construction of a two vector adenoviral system that causes the recipient cell, after its co-infection with both viruses, to become a retrovirus producing cell and spread a high-titer retroviral vector to neighboring cells [23].

\section{Tissue Targeting}

Adenoviruses are more specifically used as vehicles to administer targeted gene therapy, in the form of recombinant DNA or proteins [24]. Adenoviruses are taken up by epithelium-derived cell types, which makes the adenoviral vectors suitable for tumors but less so for hematologic malignancies. In addition, this natural tropism makes it impossible to control the systemic delivery of adenovirus in vivo. Until recently, the mechanism of binding and internalization of adenoviruses was not known. Studies on cell receptors revealed that the CAR plays a crucial role in the infection of human cells, many of which express this $46-\mathrm{kDa}$ membrane glycoprotein [25]. After anchoring at the CAR by virtue of the knob domain (the CAR ligand domain at the end of the capsid protein of the virus), the adenoviruses achieve internalization through interaction of the capsid penton protein with integrins $\alpha v \beta 3$ and $\alpha v \beta 5$ present on the target cells [26]. Studies using cells not expressing $\alpha v$ integrins indicated that the presence of these integrins is obligatory for viral integration [27]. The identification of the mechanism of adhesion and uptake of adenovirus provided important leads for retargeting adenoviral vectors to different cell receptors.

\section{Improved Adenovirus Vectors by the Regulation of Promoter Activity}

To set off transcription of the induced transgene, this DNA sequence has to have a promoter gene in front (upstream) on the DNA strand. Very promising preclinical studies have concentrated on regulation of the promoter. One of the common promoters for adenovirus transmitted genes is the promoter for cytomegalovirus, which can start the reading of the transgene at a high rate. The use of tumor or organ specific promoters, such as the ones that have already proven to be effective in animal models, will soon be investigated in phase I clinical studies [28]. In countries like China, oncolytic adenoviruses are being used for cancer treatment [29]. The limitations of these promoters are their need for a specific antigen expressed by the targeted tumor or organ tissue.

\section{Disadvantages of Adenoviral Gene Therapy}

One of the major effects of using adenoviruses in gene therapy is the hepatotoxicity and multiple organ failure. Though this is a serious concern, scientists are using specific modifications on the fibrous proteins of the virus to target adenoviruses to certain cell types, and hence to limit these effects [30].

Another major disadvantage is the accidental intake of the virus. It was found that respiratory infection and gastrointestinal tract infections are caused due to the inhalation and oral contact, respectively. The infection may either be acute or chronic. Acute infections are caused due to the lytic cycle that the virus undergoes as it enters the human epithelial cells, eventually leading to symptoms [31]. However the chronic infection usually infects the lymphoid tissues and causes no external symptoms. There exists another type of interaction, which is oncogenic within rodents, when an adenovirus integrates its own DNA into the host DNA and produces E1A proteins, hence altering transcription, deregulating apoptosis and forming malignant tumors by activating ras or $\mathrm{c}$-src signaling, polyoma middle $\mathrm{T}$ protein [31]. But, the role of E1A proteins are not seen in humans, as adenovirus sequences have not been found in human tumor cells, despite the high infection rates in the human population [31].

\section{Conclusions}

At present, adenoviruses are the most effective vectors in gene therapy. It is generally accepted that the major impediment to the successful application of gene therapy for the treatment of a range of diseases is not a paucity of therapeutic genes but the lack of an efficient non-toxic gene delivery system. While approaches using synthetic vectors are being developed, three of the most efficient gene delivery systems appropriate for clinical application which are currently available are based on virus vectors. Having evolved to deliver their genes to target cells, viruses can be easily manipulated to express therapeutic genes and the choice of virus type depends largely on the target cell and on the requirement for either transient or long term transgene expression. The stage is now set for the design and clinical application of genetically modified adenovirus vectors with specific targeting attributes.

\section{REFERENCES}

[1] Martin, Malcolm A.; Knipe, David M.; Fields, Bernard N.; Howley, Peter M.; Griffin, Diane; Lamb, Robert . Fields' virology. Philadelphia: Wolters Kluwer Health/Lippincott Williams \& Wilkins 2007; pp. 2395.

[2] Sandra G Gompf, MD, FACP, FIDSA; Chief Editor: Burke A Cunha, MD. Adenoviruses. WebMD LLC. 2012; 14 Nov, 2012.

[3] Kay MA, Holterman AX, Meuse L. Long-term hepatic adenovirus-mediated gene expression in mice following CTLA4Ig administration. Nat Genet 1995; 11:191-197.

[4] Yang Y, Li Q, Ertl HC. Cellular and humoral immune responses to viral antigens create barriers to lung-directed 
gene therapy with recombinant adenoviruses. J Virol 1995; 69:2004- 2015.

[5] Walsh, MP, et al. Computational analysis of two species C human adenoviruses provides evidence of a novel virus. J. Clin. Micobiol. 2011; 49:3482-3490.

[6] Bhuminder Singh. Retooling for Human Gene Therapy: New and Improved Adenoviral Vectors. Medicine. Aug 20, 2014.

[7] Yang Y, Wilson JM. Clearance of adenovirus-infected hepatocytes by MHC class I-restricted CD4+ CTLs in vivo. J Immunol 1995; 155:2564-2570.

[8] Roth JA, Cristiano RJ. Gene therapy for cancer: What have we done and where are we going? J Natl Cancer Inst 1997; 89:21-39.

[9] Schiedner G, Morral N, Parks RJ. Genomic DNA transfer with a high-capacity adenovirus vector results in improved in vivo gene expression and decreased toxicity. Nat Genet 1998; 18:180-183.

[10] Zoltick PW, Chirmule N, Schnell MA. Biology of E1-deleted adenovirus vectors in nonhuman primate muscle. $\mathrm{J}$ Virol 2001; 75:5222-5229.

[11] Bramson JL, Graham FL, Gauldie J. The use of adenoviral vectors for gene therapy and gene transfer in vivo. Curr Opin Biotechnol 1995; 6:590-595.

[12] Paielli DL, Wing MS, Rogulski KR. Evaluation of the biodistribution, persistence, toxicity and potential of germ-line transmission of a replication competent human adenovirus following intraprostatic administration in the mouse. Mol Ther 2000; 1:263-274.

[13] Hunt KK, Deng J, Liu TJ. Adenovirus mediated overexpression of the transcription factor E2F-1 induces apoptosis in human breast and ovarian carcinoma cell lines and does not require p53. Cancer Res 1997; 57:4722-4726.

[14] Shinoura N, Koike H, Furitu T. Adenovirus mediated transfer of caspase 8 augments cell death in gliomas implication for gene therapy. Hum Gene Ther 2000; 11:1123-1137.

[15] Yoshida K, Nishizaki M, Hunt KK. Combination therapy of adenovirus mediated overexpression of E2F-1 transcription factor and chemotherapy enhances growth suppression in breast cancer cells. Proc Am Assoc Cancer Res 2001; 42:855.

[16] Steinman RM. The dendritic cell system and its role in immunogenicity. Annu Rev Immunol 1991; 9: 271-296.

[17] Rissoan MC, Soumelis V, Kadowaki N. Reciprocal control of T helper cell and dendritic cell differentiation. Science 1999; 283:1183-1186.

[18] Teramato S, Ishii T, Matsuse T. Crisis of adenoviruses in human gene therapy. Lancet 2000; 355:1911-1912.

[19] R Alba1, A Bosch, M Chillon. Gutless adenovirus: last-generation adenovirus for gene therapy. Gene Therapy 2005; 12: S18-S27.
[20] Amalfitano A, Hauser MA, Hu H. Production and characterization of improved adenovirus vectors with the E1, E2b, and E3 genes deleted. J Virol 1998; 72:926-933.

[21] Grimm D, Kern A, Rittner K. Novel tools for production and purification of recombinant adenoassociated virus vectors. Hum Gene Ther 1998; 9:2745-2760.

[22] Fisher KJ, Kelley WM, Burda. A novel adenovirus-adeno-associated virus hybrid vector that displays efficient rescue and delivery of the AAV genome. Hum Gene Ther 1996; 7:2079- 2087.

[23] Bilbao G, Feng M, Rancourt C. Adenoviral/retroviral vector chimeras: a novel strategy to achieve high-efficiency stable transduction in vivo. FASEB J 1997; 11:624-634.

[24] Thacker, E. E.; Nakayama, M.; Smith, B. F.; Bird, R. C.; Muminova, Z.; Strong, T. V.; Timares, L.; Korokhov, N.; O'Neill, A. M.; De Gruijl, T. D.; Glasgow, J. N.; Tani, K.; Curiel, D. T. "A genetically engineered adenovirus vector targeted to CD40 mediates transduction of canine dendritic cells and promotes antigen-specific immune responses in vivo". Vaccine. 2009; 27(50): 7116-7124.

[25] Eugene Wu, Sunia A. Trauger, Glen R. Nemerow. Membrane Cofactor Protein Is a Receptor for Adenoviruses Associated with Epidemic Keratoconjunctivitis. J Virol. Apr 2004; 78(8): 3897-3905.

[26] Wickham, T. J., P. Mathias D, A. Cheresh, G. R. Nemerow. Integrins $\alpha v \beta 3$ and $\alpha v \beta 5$ promote adenovirus internalization but not virus attachment. Cell 1993; 73:309-319.

[27] Pramod P. Naranatt, Shaw M. Akula, Christopher A. Zien, Harinivas H. Krishnan, Bala Chandran. Kaposi's Sarcoma-Associated Herpesvirus Induces the Phosphatidylinositol 3- Kinase PKC- $\zeta$-MEK-ERK Signaling Pathway in Target Cells Early during Infection: Implications for Infectivity. JVirol. Jan 2003; 77(2): 1524-1539.

[28] Richards CA, Austin EA, Huber BE. Transcriptional regulatory sequences of carcinoembryonic antigen: identification and use with cytosine deaminase for tumor-specific gene therapy. Hum Gene Ther 1995; 6:881-893.

[29] Pandha, K. J. Harrington; edited by Richard G. Vile, Hardev. Viral therapy of cancer. Hoboken, N.J.: Wiley. 2008; pp. 1-13.

[30] Xin, K. Q.; Sekimoto, Y.; Takahashi, T.; Mizuguchi, H.; Ichino, M.; Yoshida, A.; Okuda, K.. "Chimeric adenovirus $5 / 35$ vector containing the clade $\mathrm{C}$ HIV gag gene induces a cross-reactive immune response against HIV". Vaccine. 2007; 25 (19): 3809-3815.

[31] Virology: Adenovirus. MicrobiologyBytes 2010. 14 Nov, 2012.

http://www.microbiologybytes.com/virology/Adenoviruses.h tml 